\section{POLARISATION OF LIGHT}

$$
\text { I. }
$$

I IGHT is said to be polarised when it presents cer1 tain peculiarities, hereafter to be described, which it is not generally found to possess. These peculiarities, although very varied in their manifestations, have one feature in common, viz. that they cannot be detected by the unassisted eye; consequently, special instrumental means are required for their investigation.
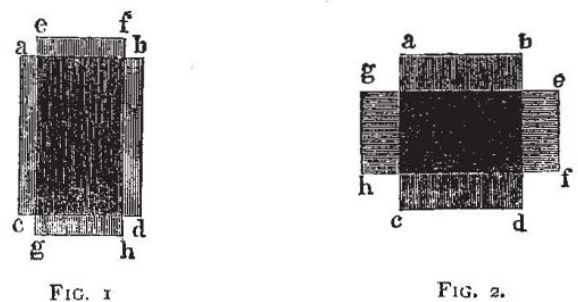

The origin and meaning of the term polarisation will be better understood when some of the phenomena have been witnessed or described, than beforehand, and I therefore postpone, for the present, an explanation of it.

The subject of polarisation may be approached by either of two roads, the experimental or the theoretical. The theoretical method, which proceeds upon the principles of the Wave Theory of Light, is remarkably complete and explicit; so much so that it not only connects.

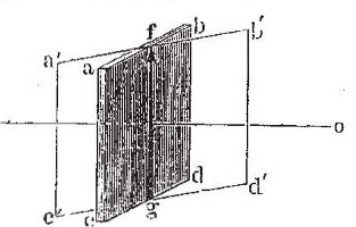

FIG. 3 .

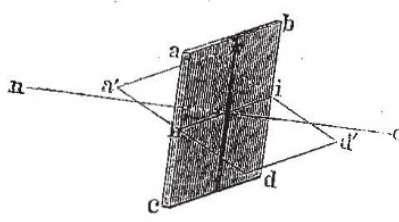

FIG. 4. together many very diversified phenomena, but even, in some cases, has suggested actual prediction. But inasmuch as the theory without experimental facts would be little better than a study of harmony without practical music, it will be best to begin with experiment.

It was stated above that certain instrumental means were requisite for detecting polarisation. Now there are various processes, some occurring in the ordinary course of natural phenomena, others due to instrumental appli-

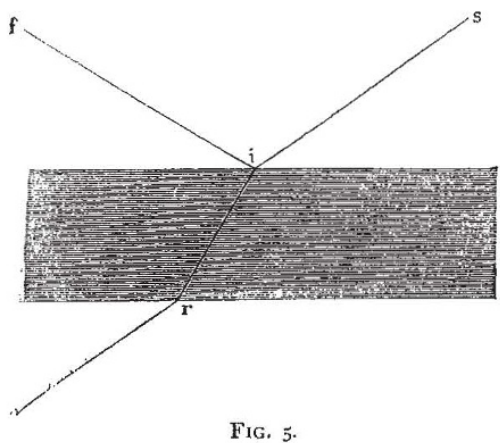

ances, whereby a ray of light may be brought into the condition in question, "or polarised." And it is a fact both curious in itself and important in its applications, that any one of these processes (not necessarily the same as that used for polarising) may be used also as a means of examining whether the ray be in that condition or not. This latter process is called " analysation." When two instruments, whether of the same or of difterent kinds are used, they are called respectively the "polariser " and the " analyser ;" and the two together are included under the general name of "polariscope."

The four principal processes by means of which a ray of light may be polarised are, reflexion, ordinary refraction, double refraction, and scattering by small particles. These methods will be considered in order; but before doing so, it will be convenient to describe the phenomena of polarisation as exhibited by some instrument tolerably simple in its action and of easy manipulation. For such a purpose a plate of crystal called tourmalin will perhaps serve better than any other to begin with.

Tourmalin is a crystal of which there are several varie-

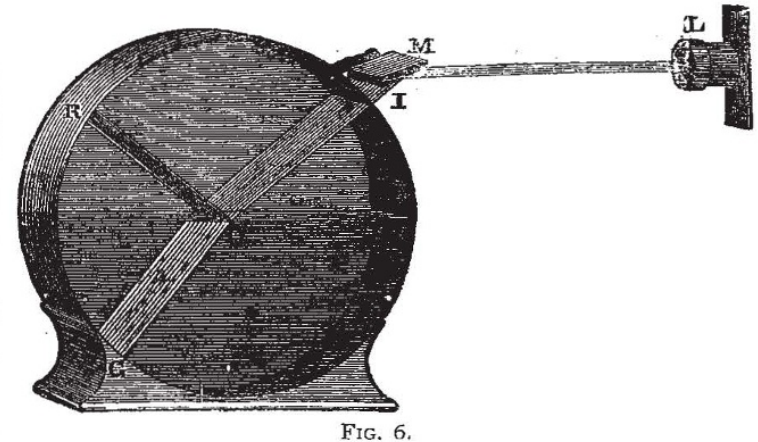

ties, differing only in colour. Very dark specimens generally answer the purpose well, excepting that it is difficult to cut them thin enough to transmit much light. Red, brown, or green specimens are usually employed; the blue are for the most part optically unsuitable. Some white, or nearly white, specimens are very good, and may be cut into thicker plates without loss of light.

If we take a plate of tourmalin cut parallel to a particular direction within the crystal called the optic axis (the nature and properties of which will be more particularly exp!ained hereafter), and interpose it in the path of a beam of light at right angles to the direction of the beam, the
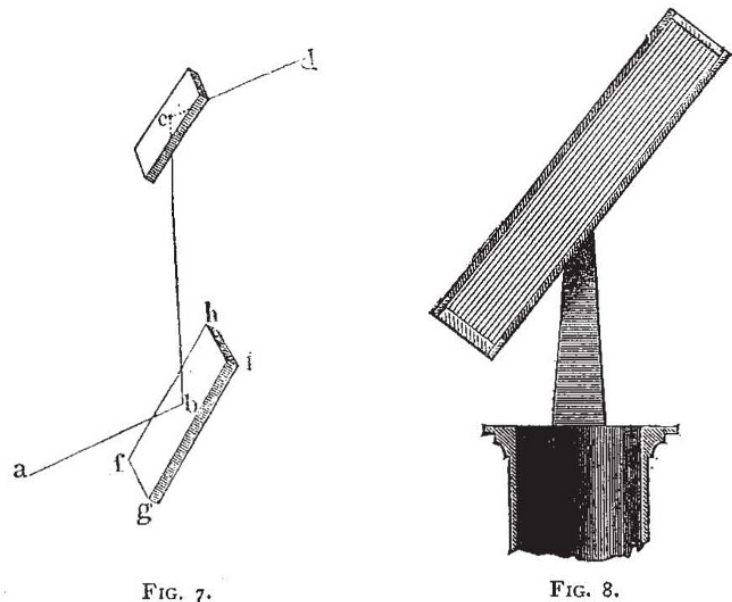

FIG. 8.

only effect perceptible to the unassisted eye will be a slight colouring of the light after transmission, in consequence of the natural tint of the particular piece of crystal. But if we examine the transmitted beam by a second similar plate of tourmalin placed parallel to the former, the following effects will be observed. When the two plates are similarly placed, i.e. as if they formed one and the same block of crystal, or as it is technically expressed, with their optic axes parallel, we shall perceive only, as before, the colouring of the light due to the tints of the two plates. But if either of the plates be then turned round in its own plane, so as always to 
remain perpendicular to the beam, the light will be cbserved to fade gradually, until, when the moving plate has been turned through a right angle, the light becomes ccmpletely extinguished. If the turning be continued bcyond the right angle, the light will begin to revive, and when a second right angle has been completed, the light will be as bright as at the outset. In Figs. I and 2 a, b, c, d, e , f, g, h represent the two plates; in Fig. I the two plates are supposed to be in the first position; in Fig. 2 the plate $e, f, g, h$ has been turned through a right angle. Of the parts which overlap, the shading in Fig. I represents the deepened colour due to the double thickness of the crystal ; in Fig. 2 it indicates the complete extinction of the light. The same alternation of brightness and extinction will continue for every right angle through which the moving plate is turned. Now it is to be observed that this alternation depends only upon the angle through which one of the crystals has been turned, or, as it is usually stated, upon the relative angular position of the two crystals. Either of them may be turned, and in either direction, and the same sequence of effect will always be produced. But if the pair of plates be turned round bodily together no change in the brightriess of the light will be made. It follows, therefore, that a ray of ordinary light possesses the same properties all round, or as it may be described, in more technical language, a ray of ordinary light, is symmetrical in respect of its properties about its own direction. On the other hand a ray of light, after traversing a plate of tourmalin has properties similar, it is true, on sides diametrically opposite to one another, but dissimilar on intermediate sides or directions; the properties in question vary in fact from one angular direction to another, and pass through their phases or an entire jeriod in every angle of $x 8$ degrees. This directional character of the properties of the ray, on account of its analogy (rather loose, perhaps) to the directional character of a magnet or an electric current, stiggested the idea of polarity, and hence the condition in which the ray was found to be was called polarisation.

Having so far anticipated the regular order of things on the experimental side of the subject, it will perhaps be worth while to make a similar anticipation on the side of theory. It is considered as established that light is due to the vibrations of an elastic medium, which, in the absence of any better name, is called ether. The ether is understood to pervade all space and all matter, although its motions are affected in different ways by the molecules of the various media which it permeates. The vibrations producing the sensation of light take place in planes perpendicular to the direction of the ray. The paths or orbits of the various vibrating ethereal molecules may be of any fom consistent with the mechanical constitution of the ether; but, on the suppositions usually made, and none simpler have been suggested, the only forms possible are the straight line, the circle, and the ellipse. But in ordinary light the orbits at different points of the ray are not all similarly situated; and although there is reason to believe that in general the orbits of a considerable number of consecutive molecules may be similarly situated, yet in a finite portion of the ray there are a sufficient number of variations of situation to prevent any preponderance of average direction.

This being assumed, the process of polarisation is un derstood to be the bringing of all the orbits through. out the entire ray into similar positions. And in the case of the tourmalin plate the orbits are all reduced to straight lines, which consequently lie in one and the same plane. For this reason the polarisation produced by tourmalin, as well as by most other crystals, is called rectilinear, or more commonly, plane polarisation. This property of tourmalin may also be expressed by saying that it permits only rectilinear vibrations parallel to a particular direction determined by its own internal structure to traverse it.
Adopting this view of polarisation as affected by a plate of tourmalin, it would be interesting to ascertain the exact direction of the vibrations. And a simple experiment will go far to satisfy us on that point. The argument, as now stated at least, is perhaps based upon general considerations rather than upon strict mechanical proof; but the experimental evidence is so strong that it should not be denied a place here. Supposefor a moment that the tourmalin be so placed that the direction of vibration lies either in or perpendicular to the plane of incidence (that is, the plane containing the incident ray, and a perpendicular to the surface on which it falls at the point of incidence); then it is natural to expect that vibrations executed in the plane of incidence will be far more affected by a change in the angle of incidence than those perpendicular to that plane. In fact the angle between the direction of the vibrations and the surface upon which they impinge, will in the first case vary with the angle of incidence; but in the second case it will remain unchanged.

In Figs. 3 and $4, n$, o represents the ray of light; the arrow the direction of vibration, a, b, c, d, a', b', $c^{\prime}, d^{\prime}$, the plate in two positions, turned in the first instance about the direction of vibration, in the second about a line perpendicular to it.

Dismissing, then, the former supposition, and supposing that nothing whatever is known about the direction of vibration; then, if all possible directions be taken in succession as pivots about which to tilt or turn the second tourmalin, it will be found that for one direction the intensity of the light diminishes more rapidly with an increase of tilting (or, what is the same thing, with an increase of the angle of incidence) than for any other. And further, that for a direction at right angles to the first, the intensity of light diminishes less than for any other; while for intermediate directions the diminution of intensity is intermediate to those above-mentioned. In accordance, therefore, with what was said before, we may conclude that the vibrations are parallel to the line or pivot about which the plate was turned when the diminution of light was least.

Secondly, polarisation may be effected by reflexion. If light reflected from the surface of almost any, except metallic, bodies be examined with a plate of tourmalin, it will in general be found to show traces of polarisation? that is to say, if the plate be caused to revolve in its own plane, and the reflected rays be viewed through it, then in certain positions of the plate, the reflected light will appear less bright than in others. If the angle at which the original rays fall upon the reflecting surface be varied, it will be found that the amount of alteration in brightness of the light seen through the revolving tourmalin (or analyser) will also vary. This fact may also be expressed thus: in polarisation by reflexion, the degree of polarisation, or the amount of polarised light in the reflected rays, varies with the angle of incidence on the reflecting surface. But at a particular angle, called on that account the polarising angle, the polarisation will be a maximum. This angle (usually measured between the incident ray and the perpendicular to the reflecting surface) is not the same for all substances; in fact it varies with their refractive power according to a peculiar law, which, when stated in the technical language of science, may be thus enunciated: the tangent of the polarising angle is equal to the refractive index. Simple geometrical considerations, combined with the usual expressions for the laws of reflexion and refraction, will show that this relation between the polarising angle and the refractive index may be also expressed in the following way: If light be incident at the polarising angle, the reflected and refracted rays will be at right angles to one another.

In Fig. 5, s, $i$ represents the incident, $i, f$ the reflected, and $i, r$ the refracted ray. Then $s, i$ will be incident at the polarising angle when the angle $s, i, r^{*}$ is a right angle. 
An apparatus devised by Prof. Tyndall for experimentally demonstrating the laws of reflexion and refraction is admirably adapted for verifying this law. The following description is quoted from his Lectures on Light:- "A shallow circular vessel RIG (Fig. 6) with a glass face, half filled with water rendered barely turbid by the admixture of a little milk or the precipitation of a little mastic, is placed upon its edge with its glass face vertical. By means of a small plane reflector $M$, and through a slit I in the hoop surrounding the vessel, a beam of light is admitted in any required direction." If a little smoke be thrown into the space above the water, the paths of the incident, the reflected, and the refracted beams will all be visible. If then the direction of the incident beam be so adjusted that the reflected and the refracted beams are at right angles to one another, and a Nicol's prism be interposed in the path of the incident beam, it will be found that by bringing the vibrations alternately into and perpendicular to the plane of incidence we shall alternately cut off the reflected and the refracted ray. Thus much for the verification of the law. But not only so, if we take different fluids and for each of them in succession adjust the incident beam in the same manner, we shall only have to read off the angle of incidence in order to ascertain the polarising angle of the fluid under examination.

The polarising angle for glass is $54^{\circ} 35^{\prime}$.

Thus, in Fig. 7, let a, b be the incident, and b, $c$ the reflected ray at the first plate; $b, c$ the incident, and $c, d$ the reflected ray at the second plate; then the ray will be polarised more or less according to the angle of incidence, at $\mathrm{b}$, and will be analysed at $\mathrm{c}$.

But in accordance with the principle stated above, viz. that any process which will serve for polarising, will serve also for analysing, we may replace the analysing tourmalin by a second plate of glass (or whatever substance has been used for the first reflexion) placed parallel to the first, and in such a position as to receive the reflected ray; and if the second plate be then turned round the ray reflected from the first plate $b c$, as an axis, it will be found that at two positions of rotation (first when the plates are parallel and secondly when one of them has been turned through $180^{\circ}$ ) the light reflected from the second plate is brightest, and at two positions at right angles to the former the reflected ray is least bright. The degree of dimness at the two positions last mentioned will depend upon the accuracy with which the reflecting plates have been adjusted to the polarising angle; and when this has been completely effected, the light will be altogether extinguished.

Suppose now that the reflecting substance be, as in the case of glass, transparent. Then it will not be surprising if, when the reflected ray is polarised, the refracted ray should also exhibit traces of polarisation. And in fact every ray of ordinary light incident upon a transparent plate is partly reflected and partly refracted; the reflected ray is partially polarised, and so also is the refracted ray. This being so, if, instead of a single plate, we use a series of plates placed one behind the other, each plate will give rise to a series of reflected rays, due to successive internal reflections. The sum of all these will give the intensity and the amount of polarisation of the total reflected light. The phenomenon of these reflexions is therefore rather complicated; and the modifications due to the additional plates do not materially alter the proportion of polarised to unpolarised light. It is, however, otherwise with the refracted rays. The rays transmitted by the first plate enter the second in a state of partial polarisation, and by a second transmission undergo a further degree of polarisation. If this process be continued by having a sufficient number of plates, the ray finally emergent may have any degree of polarisation re. quired.* And it is worthy of remark that, in proportion as

* Plates of the thinnest description are the best ; two or three give good effects, but if the surfaces lie parallel and the glass be highly transparent the number may be advantageously increased to Io, or even I2. the rays become more and more polarised, so does a less and less quantity of light become reflected from the surfaces of the plates; and consequently, except in so far as light is absorbed by actual transmission through the substance of the plates, the emergent ray suffers less and less diminution of intensity by each additional plate. So that when a certain number has been attained the inten. sity received by the eye or on a screen is practically unaffected by increasing their number.

Fig. 8 is a general representation of such a pile of plates viewed edge-ways. The plates are secured in a brass frame, and the whole supported on a stand.

(To be continued.)

$$
\text { W. SPOTTISWOODE }
$$

\section{THE ROYAL, SOCIETY}

$T \mathrm{HE}$ following extracts from the Minutes of the Council of the Royal Society under the dates given, may be interesting to some of our readers :-

Jan, 26, 1860.-The President having brought undex the consideration of the Council the present scale of remuneration of the Secretaries, it "was resolved :- "That a Committee be appointed to inquire into the matter and report thereon to the Council; the Committee to consist of the President and the Treasurer, with Mr. Barlow, Mr. Bell, and Dr. Farr."

Feb. 23, 1860.-The President presented the following Report from the Committee appointed on January 26 to consider the question of the remuneration of the Secretaries.

"Your Committee beg to Report to the Council that, in performing the task which was imposed upon them, they have inquired into the duties of the Secretaries at various times, the gratuities which have been awarded to them, and the financial condition of the Society.

"They have been favoured with valuable information and opinions by former Officers of the Royal Society,Sir John Herschel, Mr. Brande, Dr. Roget, and Sir John Lubbock.

"Previous to the year I720 no regular salaries were assigned to the Secretaries, but it was customary to present them from time to time with sums varying from Iol. to $20 l$. under the name of 'Gratuities.'

"In $I 720$, on the motion of the President, Sir Isaac Newton, the Council directed that $50 \%$. should be paid to each of the two Secretaries annually. In 1732 this amount was increased to $60 l$, and in 1760 to $70 \%$. Ios. In November 1799, on the motion of the President, Sir Joseph Banks, the amount of the salaries was reconsidered by the Council, and raised to One Hundred Guineas to each Secretary, at which amount they have remained from that time to the present.

"The office of 'Foreign Secretary' originated in a legacy of $500 l$. bequeathed to the Society in I 719 by $\mathrm{Mr}$. Robert Keck, for the express purpose of remunerating a person for carrying on foreign correspondence. In 1720 the first Foreign Secretary was appointed, with a salary of $20 l$. a year, which sum has been paid, without increase, from that time to the present.

"It is the concurrent opinion of all who have the best means of knowing, that since the gratuities were last fixed in 1799 the business of the Society and the duties of the Secretaries have largely increased. The increase of Fellows and the larger income of the Society have enabled it to extend its operations. In the ten years 1790 to 1799 , 319 papers were communicated to the Society; and in the ten years 1850 to to 1859 , the number of such papers was 672 . Some of the communications are short notices for publication in the Proceedings, and it is impossible to determine precisely in what ratio the work has increased; but your Committee are disposed to believe that it is represented approximately by the above figures. 